ARTICLE

https://doi.org/10.1038/s41467-019-13192-4

\title{
Senescent cell turnover slows with age providing an explanation for the Gompertz law
}

Omer Karin ${ }^{1,3}$, Amit Agrawal ${ }^{1,3}$, Ziv Porat (10 ${ }^{2}$, Valery Krizhanovsky (1) ${ }^{1 \star}$ \& Uri Alon (1D ${ }^{1 \star}$

A causal factor in mammalian aging is the accumulation of senescent cells ( $\mathrm{SnCs}$ ). SnCs cause chronic inflammation, and removing $\mathrm{SnCs}$ decelerates aging in mice. Despite their importance, turnover rates of $\mathrm{SnCs}$ are unknown, and their connection to aging dynamics is unclear. Here we use longitudinal $\mathrm{SnC}$ measurements and induction experiments to show that SnCs turn over rapidly in young mice, with a half-life of days, but slow their own removal rate to a half-life of weeks in old mice. This leads to a critical-slowing-down that generates persistent $\mathrm{SnC}$ fluctuations. We further demonstrate that a mathematical model, in which death occurs when fluctuating SnCs cross a threshold, quantitatively recapitulates the Gompertz law of mortality in mice and humans. The model can go beyond SnCs to explain the effects of lifespan-modulating interventions in Drosophila and C. elegans, including scaling of survival-curves and rapid effects of dietary shifts on mortality.

\footnotetext{
${ }^{1}$ Department of Molecular Cell Biology, Weizmann Institute of Science, 76100 Rehovot, Israel. ${ }^{2}$ Department of Biological Services, Weizmann Institute of Science, 76100 Rehovot, Israel. ${ }^{3}$ These authors contributed equally: Omer Karin, Amit Agrawal. *email: valery.krizhanovsky@weizmann.ac.il; uri. alon@weizmann.ac.il
} 
$\mathrm{S}$ enescent cells (SnCs) accumulate with age in mice and humans in many tissues ${ }^{1-7}$, due in part to DNA damage, damaged telomeres, and oxidative stress ${ }^{5,8}$. These cells, characterized by high levels of p16 and SA- $\beta-\mathrm{Gal}^{5}$, enter permanent cell cycle arrest, and secrete a characteristic profile of molecules including pro-inflammatory signals ${ }^{9}$ and factors that slow regeneration ${ }^{9}$ (Fig. 1a). They have physiological roles in development, cancer prevention, and wound healing ${ }^{9-11}$. However, as organisms age, accumulating levels of $\mathrm{SnC}$ cause chronic inflammation and increase the risk of many age-related diseases, including osteoarthritis, neurodegeneration, and atherosclerosis $^{12-24}$.

Accumulation of SnCs is known to be causal for aging in mice: continuous targeted elimination of whole-body $\mathrm{SnCs}$ increases mean lifespan by $25 \%$, attenuates age-related deterioration of heart, kidney, and fat, delays cancer development ${ }^{25}$ and causes improvement in the above-mentioned diseases.

These studies indicate that $\mathrm{SnC}$ abundance is an important causal variable in the aging process. Despite their importance, however, the production and removal rates of $\mathrm{SnCs}$ are unknown $^{9,26}$. For example, it is unclear whether SnCs passively accumulate or if they are turned over rapidly, and if so, whether their half-life changes with age. Since turnover affects the ability of a system to respond to fluctuations, information about these rates is crucial in order to mathematically test ideas about the possible role of SnCs in the age-dependent variations in morbidity and mortality between individuals.

Here, we address this experimentally and theoretically. To understand the dynamics of SnCs, we scanned a wide class of mathematical models of SnC dynamics, and compared these models to longitudinal $\mathrm{SnC}$ trajectories ${ }^{1}$ and direct $\mathrm{SnC}$ induction experiments in mice (Fig. $1 \mathrm{~b}-\mathrm{d}$ ). The models all describe $\mathrm{SnC}$ production and removal. They differ from one another in the way that production and removal rates are affected by age and by SnC abundance. The models describe all combinations of four possible mechanisms for accumulation of SnCs (Fig 1b): (i) $\mathrm{SnC}$ production rate increases with age due to accumulation of mutations ${ }^{27}$, telomere damage, and other factors that trigger cellular senescence ${ }^{11}$, (ii) SnCs catalyze their own production by paracrine and bystander effects ${ }^{28}$, (iii) $\mathrm{SnC}$ removal decreases with age due to age-related decline in immune surveillance functions ${ }^{29}$, and (iv) SnCs reduce their own removal rate, which can be due to SnC-related signaling, such as SASP, downregulation of immune surveillance by $\mathrm{SnCs}$, SnCs saturating immune surveillance mechanisms (similar to saturation of an enzyme by its substrate), or to disruption of tissue and extracellular matrix architecture that interferes with removal.

Mechanism (iv) is distinct from mechanism (iii) because the decline in removal rate in (iv) depends on SnC abundance, rather than on age directly. Although (iv) can arise from various biological processes, we denote it for simplicity 'saturation of removal'. These four effects lead to 16 different circuits (Fig. 1b) with all combinations of whether or not each of effects (i-iv) occur. Additionally, each of the 16 models includes parameters for basal production and removal. The models have rate constants that are currently uncharacterized. We also tested models which incorporate additional non-linearities (Supplementary Note 1, Supplementary Fig. 1).

\section{Results}

SnC dynamics during ageing in mice. To find which of the model mechanisms best describes SnC dynamics, and with which rate constants, we compared the models to longitudinal data on $\mathrm{SnC}$ abundance in mice collected by Burd et al. ${ }^{1}$. SnC abundance was measured using a luciferase reporter for the expression of p16 ${ }^{\mathrm{INK} 4 \mathrm{a}}$, a biomarker for SnCs. Total body luminescence (TBL) was monitored every 8 weeks for 33 mice, from early age (8 weeks) to middle-late adulthood ( 80 weeks) (Fig. 2a).

The luciferase in these mice was introduced into one of the p16 loci, causing the mice to be heterozygous for p16, which may impair proper activation of the senescence program. We therefore

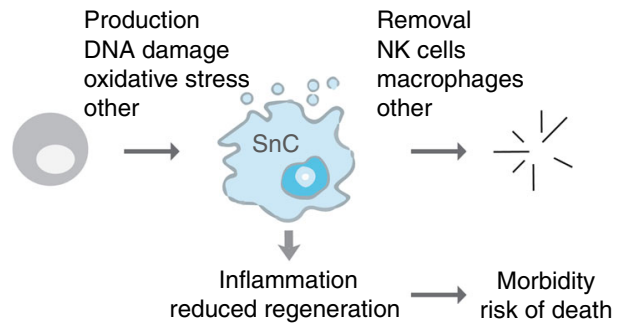

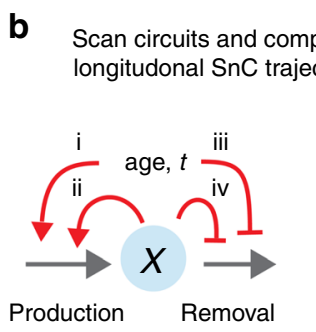

16 circuits total

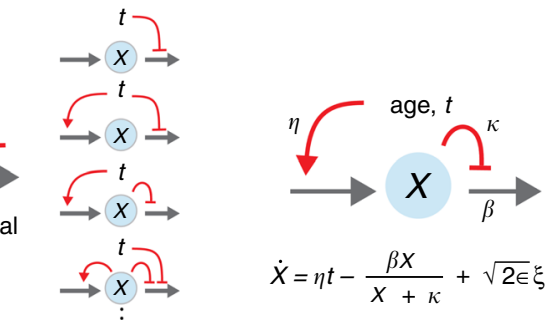

C

Minimal circuit and parameters

Fig. 1 Approach for inferring SnC dynamics throughout adulthood. a Many processes, including DNA damage and developmental and paracrine signals, lead to $\mathrm{SnC}$ production. SnCs are cleared by immune mechanisms, and secrete factors that lead to morbidity and mortality. b-d We scanned a wide class of models for SnC dynamics, and compared them to longitudinal SnC data and direct SnC perturbation experiments to establish a minimal model for SnC stochastic dynamics and determine its rate constants. In the minimal model, $\eta$ is the increase in SnC production rate with age, $\beta$ is the removal rate, $\kappa$ is the half-way saturation point for removal, and $\epsilon$ is the noise amplitude. 
a

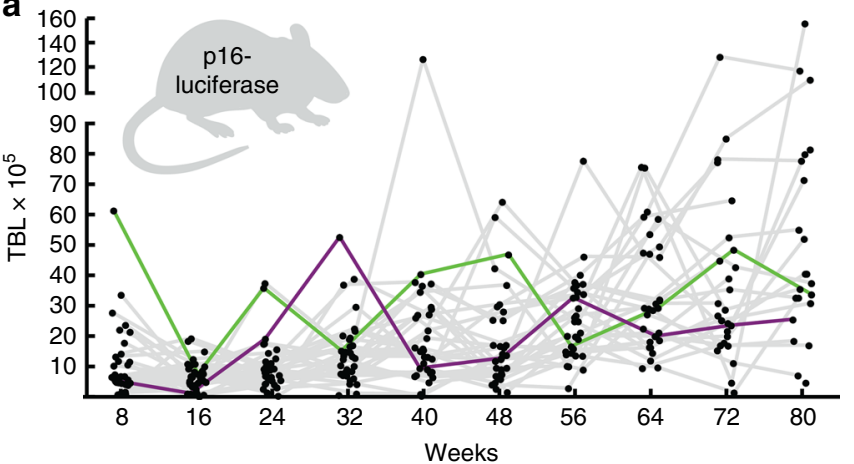

C

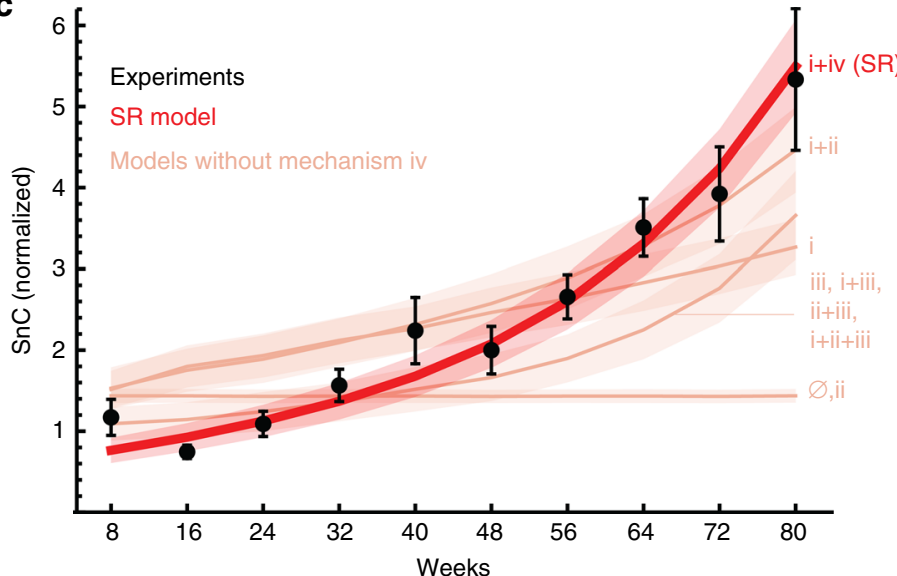

f

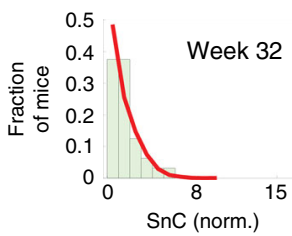

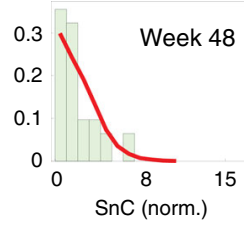

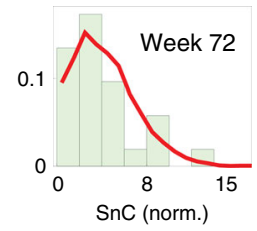

b

\begin{tabular}{ll} 
SR model & $\dot{X}=\eta t-\frac{\beta X}{X+\kappa}+\sqrt{2 \epsilon} \xi$ \\
\hline Mean & $\mu \approx \frac{\kappa \eta t+\epsilon}{\beta-\eta t}$ \\
\hline $\begin{array}{l}\text { Standard } \\
\text { deviation }\end{array}$ & $\sigma \approx \frac{\sqrt{\kappa \beta \epsilon+\epsilon}^{2}}{\beta-\eta t}$ \\
\hline Half-life & $T_{1 / 2} \approx \frac{\log 2(\epsilon+\kappa \beta)}{\beta(\beta-\eta t)}$
\end{tabular}

d

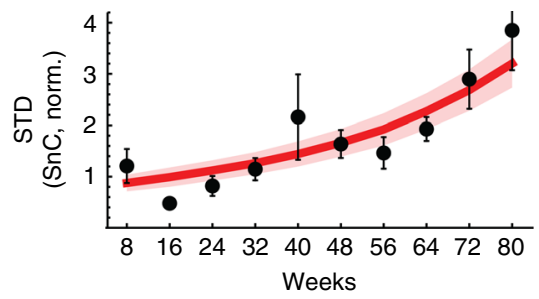

e

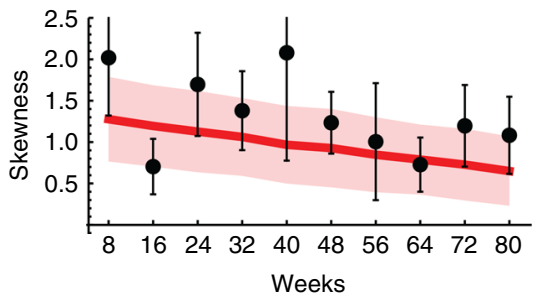

g

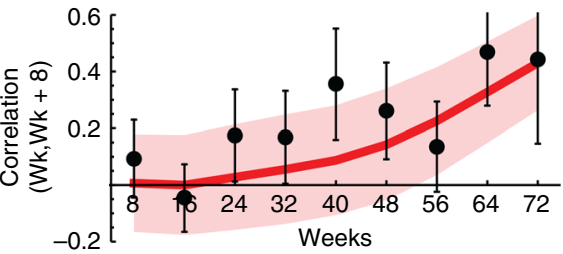

Fig. 2 Saturated-removal (SR) model captures longitudinal SnC trajectories in mice. a Total body luminescence (TBL) of p16-luciferase in mice $(n=33)$. Gray lines connect data from the same individual mice (green and purple lines are examples of individual trajectories). $\mathbf{b}$ SR model equations and their approximate analytical solutions. The SR model (red line) captures c the mean SnC abundance, d standard deviation of SnC abundance, e skewness, and $\mathbf{f}$ shape of the distributions among equal-aged individuals, and $\mathbf{g}$ correlation between subsequent measurements on the same individuals. TBL was normalized to give a mean abundance of 1 at young ages. Maximum-likelihood parameters for the SR model are: $\eta=0.15$ day $^{-1}$ year $^{-1}, \beta=0.27$ day ${ }^{-1}, \kappa=$ $1.1, \varepsilon=0.14$ day $^{-1}$. Pink lines in $\mathbf{c}$ : best-fit of all models without saturation mechanism iv, that have an age-related increase in SnCs, best-fit parameters are in Supplementary Note 1. Mean and standard error (shaded red, pink regions) are from bootstrapping. Source data are provided as a Source Data file.

also tested longitudinal measurements of SnCs based on another method. For this we obtained longitudinal data from Yamakoshi et al. ${ }^{30}$, who measured $\mathrm{SnC}$ abundance by creating a transgenic mouse model with a human p16 gene tagged with luciferase, retaining the native p16 loci. Although this dataset has much fewer mice, it shows similar dynamics to the dataset of Burd et al. ${ }^{1}$ (Supplementary Note 1, Supplementary Fig. 2), suggesting a similar underlying dynamical process.

We tested how well each model describes the longitudinal SnC trajectories of Burd et al. ${ }^{1}$ by finding the maximumlikelihood parameters for each of the 16 models, adjusting for number of parameters (Supplementary Notes 1 and 2, Supplementary Tables 1-4). A principle emerges from this comparison: in order to capture the longitudinal dynamics, the mechanism must have rapid turnover of SnCs on the timescale of a few days in young mice, and it also must include mechanism (iv), which represents a decline in removal that depends on SnC abundance rather than directly on age. The simplest model that describes the data thus has only two interactions (Fig. 1c): SnC production rate increases linearly with age (mechanism i), and SnCs slow down their own removal rate (mechanism iv). We call this model the saturating removal model (SR model), whose equation is given in Fig. 2b.

The SR model captures the accelerating rise of mean $\mathrm{SnC}$ abundance with age in the longitudinal data (Fig. 2c and Supplementary Figs. 3, 4): as SnCs accumulate, they slow their own removal, leading to even higher SnC levels. The SR model also explains the SnC variability between individuals which accelerates with age (Fig. 2d), and the $\mathrm{SnC}$ distributions among equal-aged individuals (Fig. 2e), which are skewed to the right (Fig. 2f).

Importantly, the SR model captures the fact that $\mathrm{SnC}$ fluctuations become more persistent with age, as evidenced by an increasing correlation between subsequent measurements (Fig. 2g, F-test for linear regression, $p$-value 0.0047; F-statistic 16.5): individuals with higher (or lower) than average SnC levels stay higher (or lower) for longer periods with age. This increased persistence is due to the effect of SnCs on their own removal rate. 
a
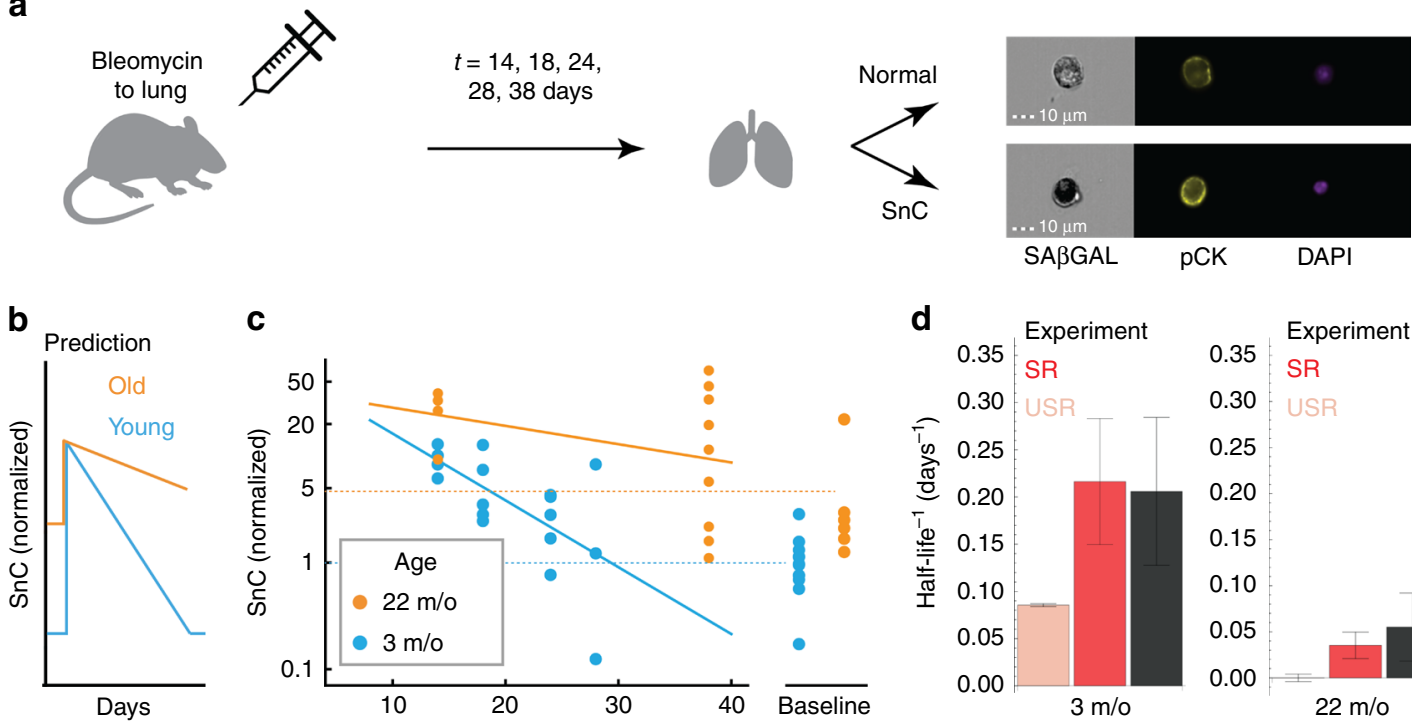

C
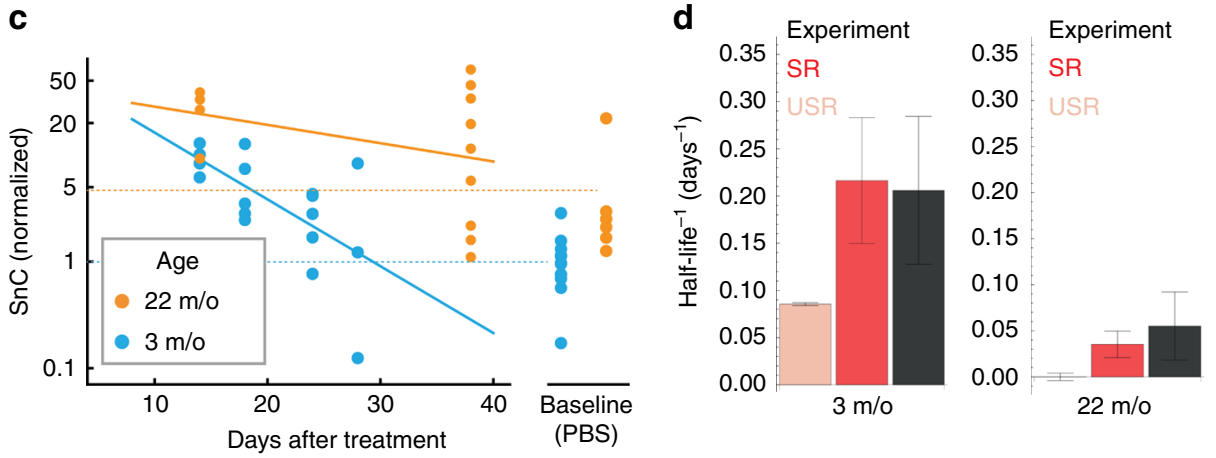

Fig. $3 \mathrm{SnC}$ half-life measurements in mice support SR model predictions. a Bleomycin or PBS was introduced by intratracheal installation to mice on day 0 . Lungs were analyzed on the indicated days thereafter. Representative images of lung cells analyzed by imaging flow cytometry show how senescent epithelial cells were identified, using SA- $\beta$-Gal, Pan-Cytokeratin (pCK), and DAPI staining. SnC removal rate was estimated by log-linear fit. b The SR model predicts that $\mathrm{SnCs}$ rapidly return to baseline in young mice and that removal is slower in old mice. c Fraction of $\mathrm{SnCs}$ in mouse lungs after treatment with bleomycin $(1.5 \mathrm{U} / \mathrm{kg}$ ). In young mice, $\mathrm{SnC}$ levels return to baseline with a half-life of about 5 days. In old mice, baseline $\mathrm{SnC}$ levels are about five-fold higher, and $\mathrm{SnC}$ removal rate is slower than in young mice . $\mathbf{d ~ S n C ~ r e m o v a l ~ r a t e s ~ ( h a l f - l i f e ~}{ }^{-1}$ ) for young and old mice (mean and standard error from bootstrapping, black) agree with the SR model predictions (red, mean and SE were calculated by bootstrapping, see the "Methods" section). The best-fit model without mechanism (iv), the USR model (mechanisms i + iii), shows a poor prediction (pink). For both ages, the USR prediction is different from the observed halflife with $p<0.01$ from bootstrapping. Source data are provided as a Source Data file.

Models without mechanism iv (saturation of removal) show a poor overall fit (pink lines in Fig. 2c, $\Delta \mathrm{BIC}>44.3$ ).

SnC lifetime is days in young mice and weeks in old mice. The maximum-likelihood parameters of the SR model (listed in the caption of Fig. 2) provide quantitative predictions for $\mathrm{SnC}$ halflives: SnC turnover is rapid in young mice, with a half-life of about $5 \pm 1$ days at 3 months of age; Turnover slows with age, so that $\mathrm{SnC}$ half-life is about $25 \pm 6$ days at 22 months.

We tested these predictions using experiments in mice by inducing SnCs and analyzing their dynamics. To induce senescence in mice lungs we used intra-tracheal bleomycin administration (Fig. 3a), a DNA-damaging agent that induces cellular senescence in the lung epithelium a few days after treatment ${ }^{5,31}$.

We quantified the fraction of senescent lung epithelial cells at different time points following bleomycin administration (Fig. 3a) using imaging flow cytometry. Epithelial SnCs were defined as cells positive for a senescent cell marker (SA- $\beta-G a l)$ and an epithelial marker (pan-Cytokeratin, pCK). This cell population was also HMGB1 nuclear negative, as expected in $\mathrm{SnCs}^{5,32}$, and previously shown ${ }^{5}$ to correspond to non-proliferative cells (negative Ki67 assay, see Supplementary Note 3, Supplementary Fig. 6).

In 3-month-old mice, SnC levels decayed with a half-life of $\tau=$ 4.7 days $\left(\tau^{-1}=0.21+/-0.07\right.$ days $\left.^{-1}\right)$ and reached their baseline level within less than a month (Fig. 3b, c), as predicted. SnC levels in young mice lungs are thus in a rapid dynamic balance of production and removal.

To test the prediction that removal slows with age (Fig. 3b), we performed the bleomycin treatment in old mice (22-month old). In these mice, the baseline level of SnCs was about five-fold higher than in young mice (Fig. 3d). SnCs decayed with a half-life of $\tau=18$ days, $\tau^{-1}=0.055+/-0.035$ days $\left.^{-1}\right)$, slower than that of young mice as predicted ( $p=0.038$ from bootstrapping, Fig. $3 b$ ).

These turnover measurements quantitatively agreed with the predictions of the SR model (Fig. 3d, Supplementary Note 4, Supplementary Fig. 7) with no additional fit. This agreement occurred despite the use of distinct $\mathrm{SnC}$ markers in the two data sets (SA- $\beta-\mathrm{Gal}$ in the bleomycin experiment vs. p16 ${ }^{\mathrm{INK} 4 \mathrm{~A}}$ luciferase in the longitudinal experiment), suggesting consistency between the measurement methods.

Our results suggest a core mechanism in which $\mathrm{SnC}$ production rate rises linearly with age, and SnCs slow their own removal (Supplementary Note 5, Supplementary Fig. 8). This slowdown of removal accelerates $\mathrm{SnC}$ accumulation with age. Slowdown of removal also amplifies fluctuations in $\mathrm{SnC}$ levels at old ages. This amplification, known as critical slowing down $\mathrm{n}^{33,34}$, results in long-lasting differences among individuals at old ages. In other words, young mice have large spare removal capacity of $\mathrm{SnC}$; old mice have much smaller spare removal capacity. This smaller removal capacity means that any addition of SnCs takes longer to remove, causing larger and more persistent variation in $\mathrm{SnC}$ levels among individuals (Fig. 2g).

The SR model quantitatively recapitulates the Gompertz law. In the remainder of the paper, we use mathematical analysis to explore the implications of these findings for the question of variability in mortality. Mortality times vary even in inbred organisms raised in the same conditions, demonstrating a nongenetic component to mortality ${ }^{35,36}$. In many species, including mice and humans, risk of death rises exponentially with age, a relation known as the Gompertz law ${ }^{37-39}$, and decelerates at very old ages. The Gompertz law has no known explanation at the cellular level. 
To connect SnC dynamics and mortality, we need to know the relationship between $\mathrm{SnC}$ abundance and risk of death ${ }^{1}$. The precise relationship is currently unknown. Clearly, $\mathrm{SnC}$ abundance is not the only cause for morbidity and mortality. It seems to be an important causal factor because removing SnCs from mice increases mean lifespan ${ }^{25}$, and adding SnCs to mice increases risk of death and causes age-related phenotypes ${ }^{23}$. We therefore explored the simple possibility that death can be modeled to occur when $\mathrm{SnC}$ abundance exceeds a threshold level $X_{\mathrm{C}}$, representing a collapse of an organ system or a tipping point such as sepsis (Fig. 4a). Thus, death is modeled as a first-passage time process, when $\mathrm{SnC}$ cross $X_{\mathrm{C}}$. We use this assumption to illustrate our approach, because it provides analytically solvable results. We also show that other dependencies between risk of death and $\mathrm{SnC}$ abundance, such as sigmoidal functions with various degrees of steepness, provide similar conclusions.

The SR model analytically reproduces the Gompertz law, including the observed deceleration of mortality rates at old ages (Fig. 4b-d, Supplementary Note 2, Supplementary Fig. 5, Supplementary Table 5). Notably, most models without both rapid turnover and slowdown of removal do not provide the Gompertz law (Supplementary Note 2). The deceleration of mortality rates at very old ages occurs in the model due to the increased persistence of $\mathrm{SnC}$ at old age. Those with high SnC have already died, whereas those with low SnC retain low SnC levels for long periods of time and avoid death. The SR model gives a good fit to the observed mouse mortality curve (Fig. 4b, c, Supplementary Note 1) using parameters that agree with the present experimental half-life measurements and longitudinal SnC data (Supplementary Note 1). Thus, turnover of days in the young and weeks in the old provides SnC variation such that individuals cross the death threshold at different times, providing the observed mortality curves.

The SR model can describe the observed increase in mean lifespan of mice in experiments in which a fraction of SnCs are continually removed (Supplementary Note 6). More generally, the SR model can address the use of drugs that eliminate SnCs, known as senolytics ${ }^{40}$. To reduce toxicity concerns, it is important to establish regimes of low dose and large inter-dose spacing ${ }^{41}$. The model provides a rational basis for scheduling senolytic drug administrations. Specifically, treatment should start at old age, and can be as infrequent as the $\mathrm{SnC}$ turnover time ( month in old mice) and still be effective (Supplementary Note 6).

We also adapted our results from the mouse data to study human mortality curves. In humans, mortality has a large nonheritable component ${ }^{42,43}$. A good description of human mortality data, corrected for extrinsic mortality, is provided by the same parameters as in mice, except for a 60 -fold slower increase in $\mathrm{SnC}$ production rate with age in the human parameter set (Fig. 4d, Supplementary Note 7, Supplementary Table 6). This slower increase in SnC production rate can be due to improved DNA maintenance in humans compared to mice ${ }^{44}$. We conclude that the critical slowing-down described by the SR model provides a possible cellular mechanism for the variation in mortality between individuals.

SR-type dynamics and ageing of Drosophila and C. elegans. The generality of the SR model suggests that it might also apply to organisms where ageing may be driven by factors other than SnCs, such as Drosophila melanogaster and C. elegans, in which lifespan variation is well-studied ${ }^{35,45}$. In these organisms, the present approach can be extended by considering $X$ as a causal factor for aging, that accumulates with age and has SR-type dynamics ${ }^{46}$, namely turnover that is much more rapid than the lifetime, increasing production and self-slowing removal. One clue for the identity of such factors may be geneexpression variations in young organisms that correlate with individual lifespan ${ }^{47-49}$, and the actions of genes that modulate lifespan $39,50-53$.

Work in C. elegans and Drosophila provides constraints to test the SR model. For example, Drosophila shows rapid switches between hazard curves when transitioned between normal and lifespan-extending diets (Fig. 4e, inset). These switches are welldescribed by the SR model, due to its rapid turnover property (Fig. 4e and Supplementary Note 8, Supplementary Fig. 9). The rapid turnover property entails that the level of $X$ can adjust after a change in any of the parameters of the model. A model without rapid turnover could not explain these results.

We further tested whether the SR model can explain the survival curves of $C$. elegans under different life-extending genetic, environmental, and dietary perturbations ${ }^{35}$. These perturbations change mean lifespan by up to an order of magnitude. The survival curves show a remarkable feature called temporal scaling: the survival curves collapse onto approximately the same curve when age is scaled by mean lifespan (Fig. 4f insets). That is, the entire distribution of death times, including its mean and standard deviation, is determined by a single parameter, which depends on the perturbation. We find that the SR model provides the shape of the survival curves, as well as their temporal scaling feature. Temporal scaling is found in the SR model by assuming that the perturbations affect the accumulation rate $\eta$ (Fig. 4f, Supplementary Note 9 and Supplementary Fig. 10A).

Temporal scaling cannot be explained by models without rapid turnover (Supplementary Fig. 10B), or by varying any other parameter except $\eta$ in the SR model. Thus, we predict loss of temporal-scaling of survival curves when a perturbation affects other SR-model parameters, such as removal rate $\beta$ or noise $\epsilon$ (Supplementary Fig. 10C, D). This prediction may apply to exceptional perturbations in which temporal scaling is not found, such as the eat-2 and nuo-6 mutations (Supplementary Fig. 10E, F). We conclude that the SR model of rapid turnover with criticalslowing down is a candidate explanation for the temporal scaling of survival curves in C. elegans.

\section{Discussion}

In this study, we propose a framework for the dynamics of SnCs based on rapid turnover that slows with age. Bleomycin-induced SnC half-life is days in young mice and weeks in old mice, causing critical slowing down, which greatly amplifies the differences between individual $\mathrm{SnC}$ levels at old age. We theoretically explore the implications of this slowdown in a model in which SnCs cause death when they exceed a threshold. The widening variation in SnC levels with age causes a mortality distribution that follows the Gompertz law of exponentially increasing risk of death. The mortality distribution of mice and humans is well-described by the SR model with the SnC half-lives measured here. Future work may test this proposed connection between SnC dynamics and mortality by experimentally measuring risk of death as a function of SnC abundance.

The rapid removal of SnCs that we observe following bleomycin-induced DNA damage is in line with studies that showed efficient removal of SnCs in vivo following liver fibrosis or induction by senescence by mutant $\operatorname{Ras}^{54-56}$. On the other hand, when senescence was induced in the skin by directly activating the cell-cycle inhibitor $p 14 A R F$, which was not associated with an increase in tissue cytokine expression or inflammation, the induced SnCs persisted in the tissue for several weeks ${ }^{57}$. 
a

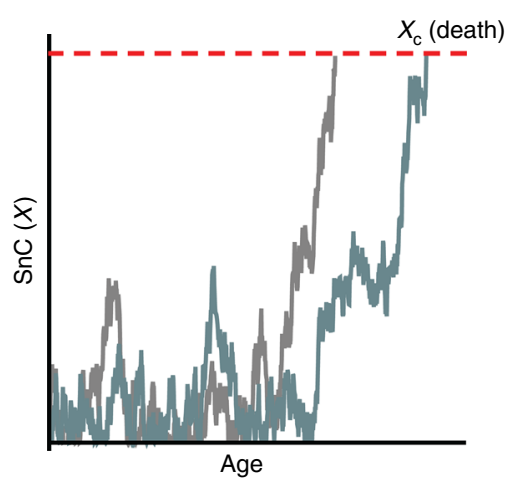

c

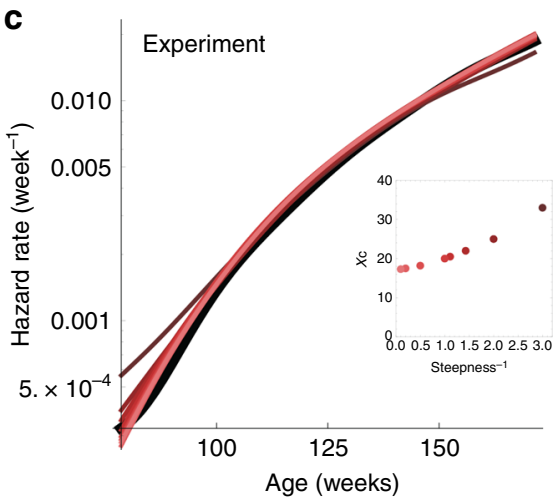

e

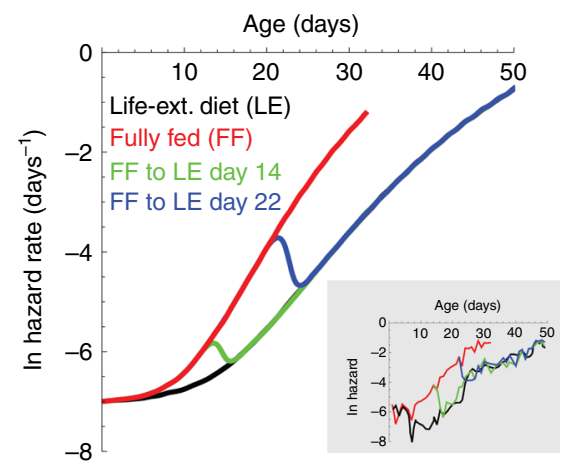

b

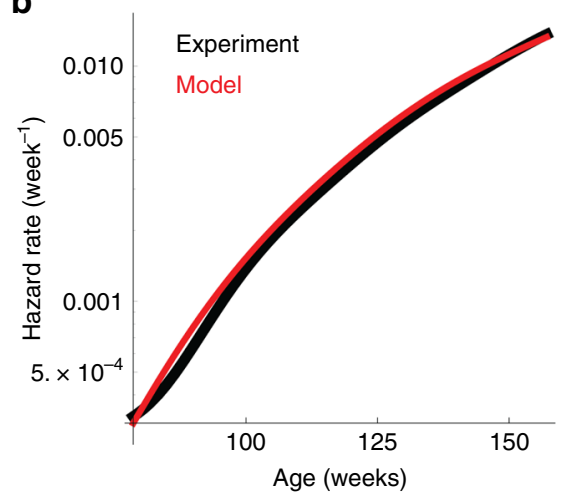

d

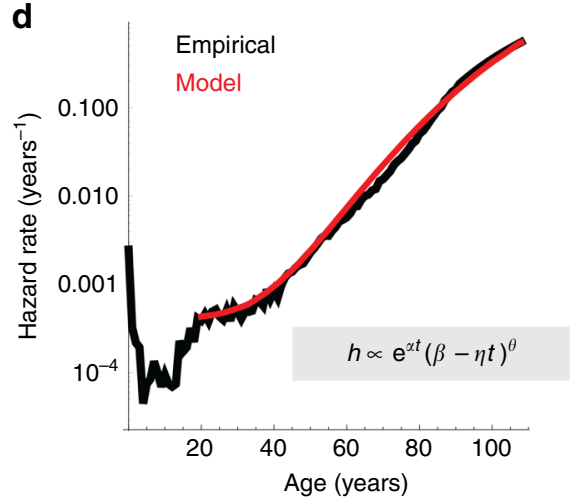

f
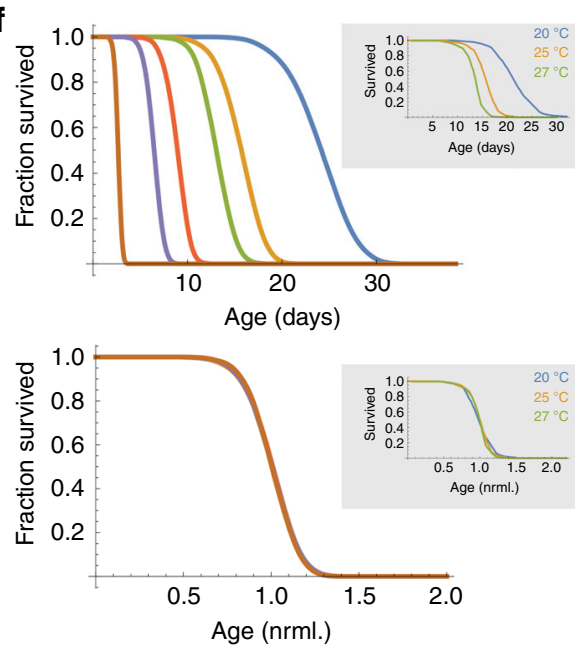

Fig. 4 SR model can explain the variability in mortality between individuals. a To model the relation between risk of death and SnC levels, we assumed a simple threshold model where death occurs when SnC abundance exceeds a critical threshold $X_{\mathrm{C}}$. b Mouse mortality (C57BL/6J mice obtained from the Mouse Phenome Database ${ }^{60}$, black line) is well fit by the SR model (red line) with parameters consistent with the data of Figs. 1, 2, with death defined when $\mathrm{SnC}$ exceed a threshold $\left(\eta=0.084\right.$ day $^{-1}$ year $^{-1}, \beta=0.15$ day $^{-1}, \kappa=0.5, \varepsilon=0.16$ day $\left.^{-1}, X_{C}=17\right)$. c Similar results are obtained by assuming a more general sigmoidal dependency between $\mathrm{SnC}$ abundance $X$ and risk of death: $h=\left(1+\mathrm{e}^{-\theta\left(X-X_{C}\right)}\right)^{-1}$. Parameters are the same as $\mathbf{b}$, except that $X_{C}$ is adjusted according to the steepness parameter $\theta$ (inset). $\mathbf{d}$ The SR model with added age-independent extrinsic mortality of $0.4 \times 10^{-3}$ year ${ }^{-1}$ (red) matches human mortality statistics ${ }^{61}$ (black). Inset: approximate analytical solution for the first passage time in the SR model shows the Gompertz law and deceleration at old ages. The parameters are similar to $\mathbf{b}$, except a $\sim 60$-fold decrease in $\eta: \eta=0.00135$ day $^{-1}$ year $^{-1}, \beta=0.15$ day $^{-1}, \kappa=0.5, \varepsilon=0.142$ day ${ }^{-1}, X_{C}=17$. e SR model describes rapid shifts in mortality when fully fed Drosophila transition to a lifespan-extending dietary intervention (LE), (inset: experimental data from Mair et al. ${ }^{45}$ ), with $\beta=1 \mathrm{~h}^{-1}, \kappa=1, \varepsilon=1 \mathrm{~h}^{-1}, \eta=0.03$ day $^{-1} \mathrm{~h}^{-1}$ and $X_{C}=15$. LE was modeled by a decrease in $\eta: \eta=0.02 \mathrm{~h}^{-1}$ day $^{-1}$ (changes in other parameters lead to similar conclusions, see Supplementary Note 8). $\mathbf{f}$ Lifespan of $C$. elegans raised at different temperatures varies by an order of magnitude, but survival curves collapse on a single curve when time is scaled by mean lifespan (inset: data from Stroustrup et al. ${ }^{35}$ ). The SR model provides scaling for perturbations that affect $\eta$, but not other parameters $\left(\beta=1 \mathrm{~h}^{-1}, \kappa=1\right.$, and $\varepsilon=1 \mathrm{~h}^{-1}, \eta=0.07 \mathrm{~h}^{-1}$ day $^{-1}, X_{\mathrm{C}}=20$, Supplementary Note 9). Source data are provided as a Source Data file. 
Clearance may thus depend on the tissue, on the method of senescence induction, and on the presence of SASP.

The present analysis of longitudinal p16 trajectories suggests that $\mathrm{SnC}$ slow down their own removal rate. This effect may be due to several mechanisms, including SASP, disruption of tissue architecture, or SnC abundance exceeding immune capacity. For the latter effect, $\mathrm{SnC}$ abundance at old age needs to be comparable to the abundance of the immune cells that remove them, which make up on the order of $0.1 \%$ of the body's cells ${ }^{58,59}$. Further research is needed to characterize these effects.

Our results suggest that treatments that remove SnCs can therefore have a double benefit: an immediate benefit from a reduced $\mathrm{SnC}$ load, and a longer-term benefit from increased SnC removal. Similarly, interventions that increase removal capacity, for example by augmenting the immune surveillance of SnC, are predicted to be an effective approach to reduce $\mathrm{SnC}$ levels. More generally, the present combination of experiment and theory can be extended to explore further stochastic processes in aging, in order to bridge between the population-level and molecular-level understanding of aging.

\section{Methods}

Stochastic model simulation. Simulations of the stochastic models were performed by using the ItoProcess function of Mathematica (V11.3), with a step size of 1 day. Negative $X$ values were avoided using a reflecting boundary condition at $X=0$. In simulations that included mortality, time of death was the first time-point where $X$ exceeded $X_{\mathrm{C}}$.

Model comparison to p16INK-luciferase measurements. We sought for each model the parameters that maximize the log-likelihood of the measured trajectories (Fig. 1a). We calculated the log-likelihood of a model $m$ with parameters $\theta$ as follows. Let $X_{i, j}$ be the measured SnC level ( $\mathrm{SnC}=\mathrm{TBL} / 9.63$ to give $\mathrm{SnC}=1$ for young mice) of mouse $j$ at time point $i$ (with $X_{0, j}=0$ ). We denote by $\operatorname{Prob}_{m, \theta, i}(a \mid b)$ the probability of reaching $\mathrm{SnC}$ level $a$ at time point $i$ given $\mathrm{SnC}$ level $b$ at time point $i-1$. We call such a step from $i-1$ to $i$ a sub-trajectory. We estimated this probability from simulations (4000 simulations for every such sub-trajectory). The $\log$-likelihood is $\operatorname{LL}(m, \theta)=\sum_{j} \sum_{i} \log \left(\operatorname{Prob}_{m, \theta, i}\left(X_{i, j} \mid X_{i-1, j}\right)\right), n=294$ subtrajectories. For each model, we sought the parameter set that maximizes the loglikelihood (see Supplementary Note 1 for more details). Confidence intervals for the best-fit parameters, as well as for estimates for $\mathrm{SnC}$ half-life, were calculated by bootstrapping (selecting mice at random with replacements). Modeling experimental noise (multiplicative noise with amplitude up to $30 \%$ ) did not affect the best-fit parameters (Supplementary Note 1). To find parameters for the model that describe both the longitudinal trajectories as well as mouse mortality statistics, we scanned the subset of parameters that fit the mortality distribution of mice. Mortality statistics of WT (C57BL/6J) mice were obtained from the Mouse Phenome Database ${ }^{60}$. Because mortality in young mice appears to be unassociated with the accumulation of $\mathrm{SnCs}^{1}$, we only considered deaths that occurred after age one year (which make up $97 \%$ of the total deaths in the dataset). We performed a comprehensive scan of values of $\beta_{0}$ and $\kappa_{0}$ and constrained $\eta, \in$ to values that give a mean and standard deviation of the simulated mortality distributions that is within $2 \%$ of the empirical values for WT mice. The critical SnC level $X_{\mathrm{C}}$ was set at $X_{\mathrm{C}}=$ 17 , which is the maximal SnC level in the Burd et al. dataset ${ }^{1}$. We then calculated maximum-likelihood parameters and confidence intervals as described above.

Population-level measures. The mean and variance at time-point $i$ are the mean and variance of $\left\{X_{i, j}\right\}_{j=1}^{N}$, where $N$ is the number of mice. Autocorrelation is the Pearson correlation of the two vectors $\left\{X_{i, j}\right\}_{j=1}^{N},\left\{X_{i+1, j}\right\}_{j=1}^{N}$. The measures were calculated in the same manner from model simulations. Typical $\mathrm{SnC}$ removal rate (half-life ${ }^{-1}$ ) for the model at a given age $i$ was estimated by $\frac{\beta}{\kappa+\bar{X}_{i}} \log (2)^{-1}$, where $\overline{X_{i}}$ is the mean SnC level at age $i$ (see Supplementary Note 4 for discussion of alternative ways to estimate $\mathrm{SnC}$ half-life). For the USR model, typical $\mathrm{SnC}$ removal rate at age $i$ is $\left(\beta_{0}-\beta_{1} i\right) \log (2)^{-1}$.

Quantification of SnCs in mouse lung epithelium. We subjected 3-month-old (young) and 22-month-old (aged) C57BL6 mice to intra-tracheal installation of 1.5 $\mathrm{U} / \mathrm{kg}$ bleomycin (Sigma) solution in PBS (or PBS as a control treatment). We euthanized the mice at $14,18,24$, and 28 days for the young mice and day 14 and day 38 for the aged mice. The quantification of senescent epithelial cells was performed as previously described ${ }^{5}$ with modification. Lung tissue was chopped into $2-5 \mathrm{~mm}$ pieces in HBSS (14025050, Gibco) on ice and incubated in the $5 \mathrm{ml}$ dissociation buffer ( $1 \mathrm{mg} / \mathrm{ml}$ Collagenase Type IV (C9263, Sigma), $0.1 \mathrm{mg} / \mathrm{ml}$ DNase I (10104159001, Roche) in HBSS) at $37^{\circ} \mathrm{C}$ for 50 min. Cells were washed with HBBS and then fixed with 4\% PFA for $5 \mathrm{~min}$. Post fixation, cells were washed and incubated with X-Gal-staining solution for $16 \mathrm{~h}$ at $37^{\circ} \mathrm{C}$. The X-Gal-staining solution consisted of $5 \mathrm{mM} \mathrm{K}_{3} \mathrm{Fe}(\mathrm{CN})_{6}, 5 \mathrm{mM} \mathrm{K} \mathrm{Fe}_{4}(\mathrm{CN})_{6} \times 3 \mathrm{H}_{2} \mathrm{O}$ and $2.5 \mathrm{mM} \mathrm{X}$ $\mathrm{Gal}$ (Inalco) in PBS at pH 5.5 containing $1 \mathrm{mM} \mathrm{MgCl}_{2}$. Post X-Gal staining the cells were fixed with fixation buffer for $30 \mathrm{~min}$ at $4{ }^{\circ} \mathrm{C}$ and washed with permeabilization buffer (00-5223-56, eBioscience, San Diego, CA). The cells were then incubated with PE-conjugated pan-cytokeratin (ab52460, Abcam) and HMGB1 (ab18256, Abcam) antibodies for an hour at $4{ }^{\circ} \mathrm{C}$. For visualization of HMGB1 antibody, we used Qdot605-labeled Goat Anti-Rabbit antibody (Q11402MP, ThermoFisher). Antibodies were diluted in the permeabilization buffer with the dilution of 1:100 of PE-conjugated pan-cytokeratin and primary HMGB1, and 1:50 of Qdot605. Before visualization, the cells were stained with DAPI and filtered through a $100 \mu \mathrm{m}$ membrane. The resulting cells were analyzed by imaging flow-cytometry using ImageStreamX mark II (Amnis, Part of EMD milipore-Merck, Seattle, WA, USA, see Supplementary Note 3 for gating strategy summary). PE staining was collected at channel 3, the DAPI at channel 7 and the Qdot605 at channel 10, in addition to the bright-field images collected at channels 1 and 9 . Analysis of the image data was performed using IDEAS 6.2 software. Cells were first gated according to their area (in $\mu \mathrm{m}^{2}$ ) and aspect ratio (ratio between width and length) of the bright field images, to eliminate debris and aggregates. Then, we gated on focused cells using the gradient RMS (which measures the sharpness quality of an image by using the average gradient of a pixel normalized for variations in intensity levels) and contrast (measures the sharpness quality of an image by detecting large changes of pixel values). Cropped cells were excluded by using the centroid X feature (the number of pixels in the horizontal axis from the upper, left corner of the image to the center of the image mask). To verify that only single cells were analyzed, cells were further gated for single nuclei using the area and aspect ratio of the nuclear image of the DAPI staining. SA-beta-Gal staining was quantified using the Mean pixel (the mean of the background-subtracted pixels) contained in the bright-field image $^{5}$. Staining of pCK was quantified using the Intensity (the sum of the background subtracted pixel values within the image) and the Max pixel (the largest value of the background-subtracted pixels contained in the image) features of the corresponding channels. To quantify staining of HMGB1 specifically, its intensity was calculated. We first gated for pCK-positive cells then for HMGB1 negative, SA- $\beta$-Gal-positive cells to quantitate the SnCs in lung epithelium. Following the method, establishment of pCK positive, SA- $\beta$-Gal-positive cells were considered senescent in further experiments. In total, the mice analyzed were 3 month-olds treated with $1.5 \mathrm{U} / \mathrm{kg}(n=17), 3$-month-olds treated with PBS $(n=$ 13), 22-month-olds treated with $1.5 \mathrm{U} / \mathrm{kg}(n=13)$, and 22 -month-olds treated with PBS $(n=6)$. We complied with all relevant ethical regulations for animal testing and research. The experiments were approved by the Weizmann IACUC committee.

Analysis of the bleomycin treatment time series. We estimated the turnover of SnCs by calculating the removal time after a perturbation with bleomycin. The removal rate is the slope of the log-linear regression model, which we fit for each experiment (with confidence intervals calculated by bootstrapping). We obtained the response time predicted by the model by bootstrapping and simulating the model after perturbation (see Supplementary Note 4 for details). Statistical significance tests were computed by bootstrapping.

Estimation of hazard and survival functions. We fit hazard and survival functions from mortality data by interpolation using the Mathematica (V11.3) function SmoothKernelDistribution and then applying the Mathematica functions HazardFunction and SurvivalFunction. For the mice survival data, the SmoothKernelDistribution was computed with a bandwidth of 80 days.

Simulation of Drosophila and C. elegans survival curves. We simulated the mortality trajectories of Drosophila and C. elegans using the SR model, by assuming a rapid turnover and saturation $\beta=1 \mathrm{~h}^{-1}, \kappa=1[\mathrm{au}]$, and also set $\varepsilon=1[\mathrm{au}]^{2} \mathrm{~h}^{-1}$ where [au] is the mean level of $X$ in young organisms. These parameters correspond to a turnover of $X$ on the order of hours. For C. elegans, to fit the survival curve obtained by Stroustrup et al. ${ }^{35}$, we set $\eta=0.07[\mathrm{au}] \mathrm{h}^{-1}$ day ${ }^{-1}$ and assumed that death occurs when $X>X_{\mathrm{C}}$ for $X_{\mathrm{C}}=20$ [au]. Similarly, to fit the Mair et al. ${ }^{45}$ data, we set the following parameters for the Drosophila simulations: $\eta=0.03[\mathrm{au}] \mathrm{h}^{-1} \mathrm{day}^{-1}$ and $X_{\mathrm{C}}=15[\mathrm{au}]$, and assumed a baseline mortality of $\ln$ hazard $=-7$ day $^{-1}$.

Reporting summary. Further information on research design is available in the Nature Research Reporting Summary linked to this article.

\section{Data availability}

The source data underlying Figs. 2a, c, d-g, 3c, 4b-d, and Supplementary Fig. 2A is available as a Source File. All other data are available from the corresponding author upon request.

\section{Code availability}

Custom code was written in Mathematica 11.3 (Wolfram) and can be found in the following link: https://github.com/omerka-weizmann/sncdynamics. The analysis file 
contains functions for simulating circuit topologies, obtaining mortality statistics and survival-curve scaling, computing log-likelihood of stochastic trajectories, and computing various summary statistics of stochastic trajectory data.

Received: 19 March 2019; Accepted: 14 October 2019;

Published online: 02 December 2019

\section{References}

1. Burd, C. E. et al. Monitoring tumorigenesis and senescence in vivo with a p16INK4a-Luciferase model. Cell 152, 340-351 (2013).

2. Liu, Y. et al. Expression of p16INK4a in peripheral blood T-cells is a biomarker of human aging. Aging Cell 8, 439-448 (2009).

3. Melk, A. et al. Expression of p16INK4a and other cell cycle regulator and senescence associated genes in aging human kidney. Kidney Int. 65, 510-520 (2004).

4. Sofue, T. et al. Tubular cell senescence in the donated kidney predicts allograft function, but not donor remnant kidney function, in living donor kidney transplantation. Am. J. Nephrol. 47, 8-17 (2018).

5. Biran, A. et al. Quantitative identification of senescent cells in aging and disease. Aging Cell 16, 661-671 (2017).

6. Herbig, U., Ferreira, M., Condel, L., Carey, D. \& Sedivy, J. M. Cellular senescence in aging primates. Science 311, 1257 (2006).

7. Zhou, S. et al. Age-related intrinsic changes in human bone-marrow-derived mesenchymal stem cells and their differentiation to osteoblasts. Aging Cell 7, 335-343 (2008).

8. Muñoz-Espín, D. \& Serrano, M. Cellular senescence: from physiology to pathology. Nat. Rev. Mol. Cell Biol. 15, 482-496 (2014).

9. He, S. \& Sharpless, N. E. Senescence in health and disease. Cell 169, 1000-1011 (2017).

10. Demaria, M. et al. An essential role for senescent cells in optimal wound healing through secretion of PDGF-AA. Dev. Cell 31, 722-733 (2014).

11. d'Adda di Fagagna, F. Living on a break: cellular senescence as a DNA-damage response. Nat. Rev. Cancer 8, 512-522 (2008).

12. Baar, M. P. et al. Targeted apoptosis of senescent cells restores tissue homeostasis in response to chemotoxicity and aging. Cell 169, 132-147.e16 (2017).

13. Chang, J. et al. Clearance of senescent cells by ABT263 rejuvenates aged hematopoietic stem cells in mice. Nat. Med. 22, 78-83 (2016).

14. Yosef, R. et al. Directed elimination of senescent cells by inhibition of BCL-W and BCL-XL. Nat. Commun. 7, 11190 (2016).

15. $\mathrm{Xu}, \mathrm{M}$. et al. Targeting senescent cells enhances adipogenesis and metabolic function in old age. eLife 4, el2997 (2015).

16. Roos, C. M. et al. Chronic senolytic treatment alleviates established vasomotor dysfunction in aged or atherosclerotic mice. Aging Cell 15, 973-977 (2016).

17. Childs, B. G. et al. Senescent intimal foam cells are deleterious at all stages of atherosclerosis. Science 354, 472-477 (2016).

18. Schafer, M. J. et al. Cellular senescence mediates fibrotic pulmonary disease. Nat. Commun. 8, 14532 (2017).

19. Ogrodnik, M. et al. Cellular senescence drives age-dependent hepatic steatosis. Nat. Commun. 8, 15691 (2017).

20. Jeon, O. H. et al. Local clearance of senescent cells attenuates the development of post-traumatic osteoarthritis and creates a pro-regenerative environment. Nat. Med. 23, 775-781 (2017).

21. Krishnamurthy, J. et al. p16INK4a induces an age-dependent decline in islet regenerative potential. Nature 443, 453-457 (2006).

22. Sagiv, A. et al. p53 in bronchial club cells facilitates chronic lung inflammation by promoting senescence. Cell Rep. 22, 3468-3479 (2018).

23. Xu, M. et al. Senolytics improve physical function and increase lifespan in old age. Nat. Med. 24, 1246-1256 (2018).

24. Musi, N. et al. Tau protein aggregation is associated with cellular senescence in the brain. Aging Cell 17, e12840 (2018).

25. Baker, D. J. et al. Naturally occurring p16(Ink4a)-positive cells shorten healthy lifespan. Nature 530, 184-189 (2016)

26. López-Otín, C., Blasco, M. A., Partridge, L., Serrano, M. \& Kroemer, G. The hallmarks of aging. Cell 153, 1194-1217 (2013).

27. Enge, M. et al. Single-cell analysis of human pancreas reveals transcriptional signatures of aging and somatic mutation patterns. Cell 171, 321-330.e14 (2017).

28. Acosta, J. C. et al. A complex secretory program orchestrated by the inflammasome controls paracrine senescence. Nat. Cell Biol. 15, 978-990 (2013).

29. Aw, D., Silva, A. B. \& Palmer, D. B. Immunosenescence: emerging challenges for an ageing population. Immunology 120, 435-446 (2007).

30. Yamakoshi, K. et al. Real-time in vivo imaging of p16Ink4a reveals cross talk with p53. J. Cell Biol. 186, 393-407 (2009).
31. Aoshiba, K., Tsuji, T. \& Nagai, A. Bleomycin induces cellular senescence in alveolar epithelial cells. Eur. Respir. J. 22, 436-443 (2003).

32. Davalos, A. R. et al. p53-dependent release of Alarmin HMGB1 is a central mediator of senescent phenotypes. J. Cell Biol. 201, 613-629 (2013).

33. Podolskiy, D. et al. Critical dynamics of gene networks is a mechanism behind ageing and Gompertz law. Phys. Q-Bio. Preprint at ArXiv:150204307 (2015).

34. Scheffer, M. et al. Early-warning signals for critical transitions. Nature 461, 53-59 (2009).

35. Stroustrup, N. et al. The temporal scaling of Caenorhabditis elegans ageing. Nature 530, 103-107 (2016)

36. Finch, C. \& Kirkwood, T. B. L. Chance, Development, and Aging (Oxford University Press, 2000).

37. Gompertz, B. On the nature of the function expressive of the Law of Human Mortality, and on a new mode of determining the value of life contingencies. Philos. Trans. R. Soc. Lond. 115, 513-583 (1825).

38. Kirkwood, T. B. L. Deciphering death: a commentary on Gompertz (1825) 'On the nature of the function expressive of the law of human mortality, and on a new mode of determining the value of life contingencies'. Philos. Trans. R. Soc. B 370, 20140379 (2015).

39. Johnson, T. E. Increased life-span of age-1 mutants in Caenorhabditis elegans and lower Gompertz rate of aging. Science 249, 908-912 (1990).

40. Ovadya, Y. \& Krizhanovsky, V. Strategies targeting cellular senescence. J. Clin. Invest. 128, 1247-1254 (2018).

41. Kirkland, J. L., Tchkonia, T., Zhu, Y., Niedernhofer, L. J. \& Robbins, P. D. The clinical potential of senolytic drugs. J. Am. Geriatr. Soc. 65, 2297-2301 (2017)

42. Kaplanis, J. et al. Quantitative analysis of population-scale family trees with millions of relatives. Science 360, 171-175 (2018).

43. Ruby, J. G. et al. Estimates of the heritability of human longevity are substantially inflated due to assortative mating. Genetics 210, 1109-1124 (2018).

44. Hart, R. W. \& Setlow, R. B. Correlation between deoxyribonucleic acid excision-repair and life-span in a number of mammalian species. Proc. Natl Acad. Sci. USA 71, 2169-2173 (1974).

45. Mair, W., Goymer, P., Pletcher, S. D. \& Partridge, L. Demography of dietary restriction and death in Drosophila. Science 301, 1731-1733 (2003).

46. Kikis, E. A., Gidalevitz, T. \& Morimoto, R. I. Protein homeostasis in models of aging and age-related conformational disease. In Protein Metabolism and Homeostasis in Aging, Vol. 694 (ed. Tavernarakis, N.) 138-159 (Springer US, 2010).

47. Rea, S. L., Wu, D., Cypser, J. R., Vaupel, J. W. \& Johnson, T. E. A stresssensitive reporter predicts longevity in isogenic populations of Caenorhabditis elegans. Nat. Genet. 37, 894-898 (2005).

48. Wu, D., Rea, S. L., Yashin, A. I. \& Johnson, T. E. Visualizing hidden heterogeneity in isogenic populations of C. elegans. Exp. Gerontol. 41, 261-270 (2006).

49. Casanueva, M. O., Burga, A. \& Lehner, B. Fitness trade-offs and environmentally induced mutation buffering in isogenic C. elegans. Science 335, 82-85 (2012).

50. Kenyon, C. J. The genetics of ageing. Nature 464, 504 (2010).

51. Kenyon, C., Chang, J., Gensch, E., Rudner, A. \& Tabtiang, R. A C. elegans mutant that lives twice as long as wild type. Nature 366, 461 (1993).

52. Gems, D. \& Partridge, L. Genetics of longevity in model organisms: debates and paradigm shifts. Annu. Rev. Physiol. 75, 621-644 (2013).

53. Clancy, D. J. et al. Extension of life-span by loss of CHICO, a Drosophila insulin receptor substrate protein. Science 292, 104-106 (2001).

54. Xue, W. et al. Senescence and tumour clearance is triggered by p53 restoration in murine liver carcinomas. Nature 445, 656 (2007).

55. Krizhanovsky, V. et al. Senescence of activated stellate cells limits liver fibrosis. Cell 134, 657-667 (2008).

56. Kang, T.-W. et al. Senescence surveillance of pre-malignant hepatocytes limits liver cancer development. Nature 479, 547-551 (2011).

57. Tokarsky-Amiel, R. et al. Dynamics of senescent cell formation and retention revealed by p14ARF induction in the epidermis. Cancer Res. 73, 2829-2839 (2013).

58. Flindt, R. Amazing Numbers in Biology (Springer-Verlag, 2006).

59. Sender, R., Fuchs, S. \& Milo, R. Revised estimates for the number of human and bacteria cells in the body. PLoS Biol. 14, e1002533 (2016).

60. Grubb, S. C., Bult, C. J. \& Bogue, M. A. Mouse phenome database. Nucleic Acids Res. 42, D825-D834 (2014).

61. Barbieri, M. et al. Data resource profile: the human mortality database (HMD). Int. J. Epidemiol. 44, 1549-1556 (2015).

\section{Acknowledgements}

U.A. is the incumbent of the Abisch-Frenkel chair. O.K. is an Azrieli Fellow. This work was supported by grants to V.K. from the European Research Council under the European Union's FP7 and H2020 Programs and the Israel Science Foundation. 


\section{Author contributions}

Conceptualization, O.K., U.A. and V.K.; Planning and setup of mouse experiments, A.A., V.K., Execution of experiments: A.A., Analysis of ImageStreamX data, A.A, Z.P.; Derivation of theory, O.K. and U.A.; Data analysis and modeling, O.K.; Writing manuscript, O.K. and U.A.; Review and editing: O.K., A.A., U.A. and V.K.

\section{Competing interests}

The authors declare no competing interests.

\section{Additional information}

Supplementary information is available for this paper at https://doi.org/10.1038/s41467019-13192-4.

Correspondence and requests for materials should be addressed to V.K. or U.A.

Peer review information Nature Communications thanks the anonymous reviewer(s) for their contribution to the peer review of this work. Peer reviewer reports are available.

Reprints and permission information is available at http://www.nature.com/reprints
Publisher's note Springer Nature remains neutral with regard to jurisdictional claims in published maps and institutional affiliations.

(c) (i) Open Access This article is licensed under a Creative Commons Attribution 4.0 International License, which permits use, sharing, adaptation, distribution and reproduction in any medium or format, as long as you give appropriate credit to the original author(s) and the source, provide a link to the Creative Commons license, and indicate if changes were made. The images or other third party material in this article are included in the article's Creative Commons license, unless indicated otherwise in a credit line to the material. If material is not included in the article's Creative Commons license and your intended use is not permitted by statutory regulation or exceeds the permitted use, you will need to obtain permission directly from the copyright holder. To view a copy of this license, visit http://creativecommons.org/ licenses/by/4.0/.

(C) The Author(s) 2019 